\title{
Linguodidactica KKU
}

DOI: 10.15290/lingdid.2021.25.04

\author{
mgr Urszula Kochanowska \\ Uniwersytet w Białymstoku \\ Wydział Filologiczny, Katedra Leksykologii i Pragmalingwistyki \\ tel. (85) 7457526 \\ e-mail: u.kochanowska@uwb.edu.pl \\ ORCID ID: https://orcid.org/0000-0002-8038-8636
}

\section{LES TECHNIQUES DE TRADUCTION DES URBANONYMES SUR L'EXEMPLE DES ROMANS POLICIERS POLONAIS ET LEURS TRADUCTIONS EN FRANÇAIS}

\section{ABSTRAKT \\ TECHNIKI TŁUMACZENIA URBANONIMÓW NA PRZYKŁADZIE POLSKICH POWIEŚCI KRYMINALNYCH I ICH FRANCUSKICH TKUMACZEŃ}

Uważa się powszechnie, że nazwy własne nie podlegają tłumaczeniu. Autorkę interesuje transferowanie urbanonimów w przetłumaczonych na język francuski dwóch polskich powieściach kryminalnych, kłórych akcja toczy się we współczesnej Warszawie. Analiza została oparta na technikach łłumaczenia nazw własnych K. Hejwowskiego $(2004,2015)$. Wyodrębniono techniki dominujące w różnych kategoriach urbanonimów (transfer prosty, transfer z modyfikacją graficznq, tłumaczenie). W przypadku nazw ulic, alej i placów pochodzących od nazwiska tłumacze stosują neutralizację fleksyjnq. Inną często stosowaną techniką jest dodawanie kwalifikatorów rue ('ulica') i quartier ('osiedle') do nazw ulic i osiedli. W języku polskim często się je pomija, co w przypadku nazw ulic jest niedopuszczalne w języku francuskim. Zaobserwowano również techniki pozwalające na usunięcie nazwy własnej lub zastapienie jej nazwa pospolitą. Zastosowane techniki łłumaczenia urbanonimów ułatwiaja francuskiemu odbiorcy śledzenie watków powieści oraz odczytanie obcych nazw. Pozbawiają go jednak możliwości poznania nazewnictwa z innego obszaru kulturowego.

Słowa kluczowe: urbanonimy, nazwy własne, techniki łłumaczeniowe, Warszawa, powieść kryminalna

\section{ABSTRACT}

\section{TECHNIQUES OF TRANSLATING URBANONYMS DERIVED FROM POLISH CRIME NOVELS AND THEIR FRENCH TRANSLATIONS}

There is a common belief that proper names are not to be translated. The author traced the transfer of urbanonyms in French translations of two Polish crime novels set in contemporary 
Warsaw. The analysis has been based on the techniques of translating proper names by K. Hejwowski $(2004,2015)$. The dominant techniques used in various categories of urbanonyms have been distinguished (simple transfer, transfer with spelling modification, translation). In the case of street names, avenues and squares derived from surnames, translators use inflectional neutralization. Another frequent technique is to add the qualifiers rue ('street') and quartier ('district') to the names of streets and neighbourhoods. In Polish, they are often omitted, which, in the case of street names, is unacceptable in French. Moreover, several techniques allowing removing a proper name or to replace it with a common noun have also been detected. All in all, the techniques applied for translating urbanonyms make it easier for the French recipient to follow the threads of the novel and to read foreign names. However, they deprive him/her of contact with some features of foreign names' strangeness that characterize a different cultural area.

Key words: urbanonyms, proper names, translation techniques, Warsaw, crime novel

\section{Introduction}

On dit souvent que les noms propres ne provoquent pas de problèmes de traduction, car ils sont transférés du texte source sans modifications, on les exclut donc de la traduction. Cette approche explique pourquoi la traduction des noms propres n'est pas considérée comme problème dans les études théoriques, surtout anglaises, allemandes et polonaises ${ }^{1}$. Cependant, dans les dernières années, ce sujet est abordé d'une manière plus large par Krzysztof Hejwowski qui, dans Kognitywno-komunikacyjna teoria przekładu, donne une place importante à la traduction des noms propres dans le chapitre intitulé Nieprzekładalność kulturowa : "kultura to warowny obóz $»^{2}$. Dans un autre ouvrage intitulé Iluzja przektadu, il consacre un chapitre entier aux noms propres et leur traduction (Nazwy własne w tłumaczeniu) ${ }^{3}$. En outre, plusieurs chercheurs développent ce problème en analysant, dans leurs articles, la traduction des anthroponymes et des toponymes ${ }^{4}$.

1 R. Lewicki, Zagadnienia lingwistyki przekładu, Lublin 2017, s. 265-266.

2 K. Hejwowski, Kognitywno-komunikacyjna teoria przekładu, Warszawa 2004, s. 71-104.

3 K. Hejwowski, Iluzja przekładu, Katowice 2015, s. 125-179.

4 A. Cieślikowa, Jak „ocalić w tłumaczeniu” nazwy własne?, [w:] Między Oryginałem a Przekładem II. Przekład, jego tworzenie się i wpływ, M. Filipowicz-Rudek, J. Konieczna-Twardzikowa (red.), Kraków 1996; E. Skibińska, Nazwy własne we francuskim przekładzie Prawieku i innych czasów Olgi Tokarczuk, [w:] Przekładając nieprzekładalne, W. Kubiński, O. Kubińska, T.Z. Wolański (red.), Gdańsk 2000; T. Grass, La traduction comme appropriation : le cas des toponymes étrangers, « Meta » 2006, vol. 51(4); L. Waleryszak, Quelques réflexions sur la traduction des urbanonymes dans la littérature polonaise contemporaine, [in:] Traduire la ville, J. Brzozowski (réd.), Kraków 2006; J. Cook, Traduire Marseille : les repères spatiaux dans la traduction polonaise du roman Total Khéops de Jean-Claude Izzo, [in:] Challenges in Translation: Space, Culture and Linguistic Identity, R. Vasilescu, E. Nicolescu, R. Ştefan, A. Rădulescu (réd.), New 
Dans le présent article, nous aborderons les problèmes de la traduction des urbanonymes dans les romans policiers. Ce sujet nous semble important car l'action des romans policiers se déroule le plus souvent dans une ville réelle. Le récepteur d'un roman policier suit non seulement l'intrigue, mais a aussi l'occasion de connaître les endroits réels de cette ville, p.ex. : rues, quartiers, places, restaurants, cafés ou bâtiments publics. Les problèmes de la traduction des noms de ces éléments réels sont dus à leur construction, leur aspect sémantique et leurs fonctions dans le texte littéraire ${ }^{5}$. De plus, Krzysztof Hejwowski critique le manque de cohérence dans la traduction des toponymes, ainsi que de nombreuses erreurs de traduction ${ }^{6}$.

D'après Kwiryna Handke, les toponymes, se référant à l'agglomération urbaine (pl. «nazewnictwo miejskie»), comprennent des lieux de caractère varié :

- zones (p.ex. : quartiers, cités),

- voies de communication (p.ex. : rues, avenues),

- points fixes (p.ex. : places, ronds-points, etc., ainsi que des bâtiments publics) ${ }^{7}$.

Nous pouvons appeler les éléments mentionnés ci-dessus "urbanonymes". Lydia Waleryszak comprend ce terme de manière plus étroite et l'utilise seulement pour les voies de communication ${ }^{8}$. Nous, dans l'analyse qui suit, considérons comme urbanonymes les zones, les voies de communication et certains points fixes (sauf bâtiments publics).

\section{Techniques de traduction des noms propres}

Dans la traduction des textes littéraires, trois procédés principaux sont possibles : adaptation, translocation sans modification (pl. "translokacja») et traduction?. Selon Thierry Grass, il existe quelques procédés généraux pour transférer les toponymes. Le nom propre peut rester inchangé, peut être transcrit, translittéré ou traduit. Il existe aussi la possibilité de combiner ces différents procédés. Dans la majorité des cas, le transfert d'un nom propre est un em-

York 2012; N. Paprocka, Les tendances dans la traduction des toponymes dans la littérature de jeunesse: l'exemple des traductions polonaises d'Alexandre Dumas, Les Trois Musquetaires, [in:] Challenges in Translation: Space, Culture and Linguistic Identity, R. Vasilescu, E. Nicolescu, R. Ştefan, A. Rădulescu (réd.), New York 2012.

5 L. Waleryszak, Quelques réflexions..., p. 113.

6 K. Hejwowski, Iluzja przektadu..., s. 175.

7 K. Handke, Nazewnictwo miejskie, [w:] Polskie nazwy własne. Encyklopedia, E. Rzetelska-Feleszko (red.), Kraków 2005, s. 295.

$8 \quad$ L. Waleryszak, Quelques réflexions..., p. 112.

9 A. Cieślikowa, Jak „ocalić w tłumaczeniu”..., s. 314-316. 
prunt qui consiste à importer le mot tel quel, sans rien changer à la structure graphique. Quant à la traduction au sens strict, les plus courants en toponymie sont le calque et l'adaptation ${ }^{10}$.

Michel Ballard a élaboré les techniques appliquées pour traduire les noms propres de l'anglais en français. Énumérons celles qui concernent les noms des lieux à l'intérieur des villes :

a) le report - consiste à transférer le nom propre dans le texte cible :

- avec une note en bas de page,

- avec l'ajout d'un nom commun catégorisateur ou avec une explication dans le texte ;

b) la traduction plus ou moins littérale ${ }^{11}$.

Nous allons nous baser sur les techniques de traduction des noms propres élaborées par Krzysztof Hejwowski, qui s'appuie sur les techniques proposées par d'autres chercheurs, p.ex. : Jean-Paul Vinay et Jean Darbelnet, Peter Newmark ${ }^{12}$. Hejwowski présente d'abord un répertoire de six techniques, assez descriptives $^{13}$, et le développe ensuite jusqu'au nombre de $17^{14}$.

Nous en avons choisi les techniques dont nous allons nous servir comme base pour notre analyse de la traduction des urbanonymes :

a) le transfert (pl. «transfer») ${ }^{15}$ simple du nom propre dans le texte cible ;

b) le transfert avec une modification graphique ;

c) le transfert avec l'adaptation au paradigme de flexion de la langue cible ;

d) le transfert avec une explication :

- la note explicative en bas de page ;

- l'ajout du nom commun catégorisateur (rue, ville) ;

- la traduction du nom commun catégorisateur ;

- l'ajout d'un commentaire dans le texte ;

e) la traduction au moyen de l'équivalent lexical des mots ou des morphèmes faisant partie des noms propres ;

f) le remplacement du nom propre par le nom commun ;

g) la suppression du nom propre, parfois avec le contexte ${ }^{16}$.

10 T. Grass, La traduction..., p. 662-663.

11 M. Ballard, Le nom propre en traduction, Paris 2001, p. 18-38, 110-111, 135.

12 J.-P. Vinay, J. Darbelnet, Stylistique comparée du français et de l'anglais, Paris 1958; P. Newmark, A Textbook of Translation, New York 1988.

13 K. Hejwowski, Kognitywno-komunikacyjna..., s. 92-93.

14 K. Hejwowski, Iluzja przekładu..., s. 176-179.

15 La technique consistant à transférer le nom propre sans modification est donc nommée différemment par les chercheurs : pl. translokacja (A. Cieślikowa, Jak „ocalić w tłumaczeniu”...), fr. report (M. Ballard, Le nom propre...), pl. reprodukcja (K. Hejwowski, Kognitywno-komunikacyjna...), fr. emprunt (T. Grass, La traduction...), pl. transfer (K. Hejwowski, Iluzja przektadu...).

16 K. Hejwowski, Iluzja przekładu..., s. 176-179. 


\section{Corpus et méthode}

Nous avons analysé les urbanonymes dans la traduction française de deux romans policiers polonais : Uwikłanie (Les Impliqués) et Podpalacz (Pyromane)17, œuvres dont l'action se déroule à Varsovie, la capitale de la Pologne, au début du XXI siècle. Nous avons relevé 101 urbanonymes (ayant au total 295 occurrences). Les exemples récupérés comprennent : quartiers, cités, rues, avenues, places et ronds-points. L'analyse préliminaire nous a amenée à diviser les exemples en trois catégories :

1) quartiers et cités ;

2) rues ;

3) avenues, allées, places et ronds-points.

Grâce à l'analyse des techniques de traduction appliquées, nous allons vérifier si les traducteurs les utilisent de manière conséquente. Nous voulons aussi observer si les deux traducteurs se servent des mêmes techniques pour chaque catégorie des urbanonymes.

\section{Analyse du corpus}

Dans la plupart des cas, les urbanonymes se composent de deux segments : le nom commun et le nom propre. Le premier segment indique le type de zone ou de voie, p.ex. : quartier, rue, avenue, place, etc., c'est le "nom commun catégorisateur $»^{18}$ qui figure d'habitude avec la minuscule initiale. Le second segment est un nom propre.

(pl) plac Bankowy, (fr) place Vendôme

(pl) osiedle Chomiczówka, (fr) quartier du Marais

Cependant, les noms des quartiers et des cités fonctionnent plus souvent, aussi bien en polonais qu'en français, sans leur nom commun catégorisateur :

(pl) Chomiczówka, (fr) Le Marais.

\subsection{Quartiers et cités}

Les techniques utilisées pour traduire les noms de quartiers et de cités sont surtout :

17 Z. Miłoszewski, Uwikłanie, éd. W.A.B. 2014; Z. Miłoszewski, Les Impliqués, K. Barbarski (trad.), éd. Mirobole/Pocket 2015; W. Chmielarz, Podpalacz, éd. Marginesy 2018; W. Chmielarz, Pyromane,

É. Veaux (trad.), éd. Agullo/Livre de Poche 2018.

18 L. Waleryszak, Quelques réflexions..., p. 112. 
- le transfert simple

\begin{tabular}{|l|l|l|}
\hline \multirow{2}{*}{$(1)$} & \multicolumn{1}{|c|}{ Uwikłanie - Les Impliqués } & \multicolumn{1}{c|}{ Podpalacz - Pyromane } \\
\cline { 2 - 3 } & Ochota - Ochota & Praga - Praga (3) $)^{19}$ \\
Bielany - Bielany & Bemowo - Bemowo (2) \\
& & Bielany - Bielany \\
& & Kabaty - Kabaty \\
\hline
\end{tabular}

- le transfert avec une modification graphique

\begin{tabular}{|c|l|l|}
\hline \multirow{3}{*}{$(2)$} & \multicolumn{1}{|c|}{ Uwikłanie - Les Impliqués } & \multicolumn{1}{c|}{ Podpalacz - Pyromane } \\
\cline { 2 - 3 } & $\begin{array}{l}\text { Gocław - Goclaw (2) } \\
\text { Ursynów - Ursynow }\end{array}$ & $\begin{array}{l}\text { Ursynów - Ursynow (22) } \\
\text { Gocław - Goclaw (6) } \\
\text { Mokotów - Mokotow }\end{array}$ \\
\hline
\end{tabular}

- l'ajout du nom commun catégorisateur

\begin{tabular}{|c|l|l|}
\hline (3) & \multicolumn{1}{|c|}{ Uwikłanie - Les Impliqués } & \multicolumn{1}{c|}{ Podpalacz - Pyromane } \\
\cline { 2 - 3 } & $\begin{array}{l}\text { Żoliborz - quartier Zoliborz (4) } \\
\text { Ochota - quartier Ochota (3), quartier } \\
\text { d'Ochota } \\
\text { Bielany - quartier Bielany (2) } \\
\text { Wola - quartier Wola, quartier } \\
\text { de Wola }\end{array}$ & $\begin{array}{l}\text { Gocław - quartier de Goclaw (3) } \\
\text { Praga - quartier de Praga (2) } \\
\text { Bemowo - quartier Bemowo }\end{array}$ \\
\hline
\end{tabular}

Dans les exemples ci-dessus (3), nous observons que les traducteurs utilisent ou non la préposition de avant le nom de quartier, ce qui illustre, à notre avis, le manque d'attention de la part des traducteurs.

Dans le texte de départ, le nom du quartier est précédé seulement quatre fois des noms communs osiedle ou blokowisko qui sont traduits :

\begin{tabular}{|c|c|c|}
\hline \multirow{3}{*}{$4)$} & Uwikłanie - Les Impliqués & \multicolumn{1}{c|}{ Podpalacz - Pyromane } \\
\cline { 2 - 3 } & $\begin{array}{c}\text { osiedle Za Żelazną Bramą - quartier } \\
\text { Derrière-la-Porte-de-Fer }\end{array}$ & $\begin{array}{l}\text { blokowisko Ursynów/na Ursynowie - } \\
\text { HLM d'Ursynow (2) } \\
\text { osiedle Konstancin - quartier } \\
\text { Konstancin }\end{array}$ \\
\hline
\end{tabular}

Nous voyons aussi ci-dessus un seul exemple de la traduction littérale du nom de quartier, ce qui ne semble pas convenable, même si les éléments de ce nom

19 Le nombre d'occurrences quand il est plus grand qu'un. Les exemples sans nombre indiqué entre parenthèses n'apparaissent qu'une seule fois dans le texte. 
sont traduisibles à l'aide des équivalents lexicaux. Nous ne devrions pas traduire les noms propres authentiques. De plus, puisque c'est un nom propre composé, le traducteur l'adapte à l'orthographe des noms composés des quartiers français (Saint-Germain-des-Près, Val-de-Grâce, Arts-et-Métiers), en ajoutant les traits d'union (Derrière-la-Porte-de-Fer).

Nous avons aussi observé que parfois, le traducteur ajoute dans le texte d'arrivée un adjectif ou un complément caractérisant un quartier, ce qui pourrait peut-être aider le récepteur cible à connaître la particularité de quelques quartiers intéressants :

\begin{tabular}{|c|c|c|}
\hline (5) & Uwikłanie - Les Impliqués & Podpalacz - Pyromane \\
\cline { 2 - 3 } & $\begin{array}{l}\text { Żoliborz - charmant quartier Zoli- } \\
\text { borz (2) }\end{array}$ & $\varnothing$ \\
$\begin{array}{l}\text { Ochota - quartier dortoir Ochota } \\
\text { Wilanów - quartier chic de Wilanow } \\
\text { Wola - quartier de Wola à Varsovie- } \\
\text { ouest }\end{array}$ & \\
\hline
\end{tabular}

Certains noms propres des quartiers sont supprimés et remplacés dans le texte traduit par les noms communs ou les expressions comprenant un nom commun et un autre nom propre :

\begin{tabular}{|c|c|c|}
\hline (6) & Uwikłanie - Les Impliqués & Podpalacz - Pyromane \\
\hline & $\begin{array}{l}\text { Śródmieście - le centre-ville (9), le } \\
\text { centre } \\
\text { Praga - la rive droite (4), la rive droite } \\
\text { de la Vistule } \\
\text { Powiśle - le centre-ville, quais de la } \\
\text { Vistule } \\
\text { Żoliborz - quartier Bielany } \\
\text { Chomiczówka - rue Andersen, } \\
\text { quartier, quartier Bielany }\end{array}$ & $\varnothing$ \\
\hline
\end{tabular}

Dans le texte d'arrivée, le nom propre Śródmieście n'apparaît qu'une seule fois malgré ses dix occurrences dans le texte de départ. Le traducteur a probablement décidé de supprimer ce nom propre à cause de sa longueur, de la présence de trois signes diacritiques polonais et de la prononciation extrêmement difficile pour un lecteur français. L'autre nom propre de quartier supprimé dans la traduction, probablement pour des raisons similaires, est Chomiczów ka. Le traducteur le remplace non seulement par les noms communs, mais aussi par les noms propres suivant le contexte : quartier, rue Andersen, quartier Bielany. Au fait, la rue Andersen se trouve dans la cité Chomiczówka, qui est située dans le quar- 
tier Bielany. Presque tous les exemples de la traduction cités ci-dessus (sauf rue Andersen) illustrent l'application d'une sorte d'hyperonyme, des mots ou des expressions aidant le lecteur français à situer plus facilement le quartier dans la ville de Varsovie. Le quartier Śródmieście, se trouvant dans le centre-ville, pourrait être traduit littéralement comme Centre-Ville. Praga est situé sur la rive droite de la Vistule ; Żoliborz et Chomiczówka font partie du quartier Bielany.

\subsection{Rues}

Quant aux voies de communication, nous avons repéré un grand nombre de noms de rues qui apparaissent dans le texte source sous la forme d'un seul segment (nom propre, p.ex. : Chmielna, Belwederska, Pawińskiego) ou de deux segments (nom commun catégorisateur + nom propre, p.ex. : ulica Mokotowska, ulica Pawińskiego). Le premier type de nom a beaucoup plus d'occurrences que le second. Il est possible, dans la langue polonaise, d'exclure le nom commun et de n'employer que le nom propre : mieszkam na/przy Solskiego (' $\mathrm{j}$ 'habite sur/à côté de Solskiego'). Ce procédé est impossible en français ${ }^{20}$. Les formules mono-lexicales elliptiques existent dans la langue française, mais dans des cas particuliers dont parle Waleryszak : «Si l'on peut abréger la rue Nationale par la Nationale ou l'avenue des Champs-Élysées par les Champs-Élysées, il semble incorrect d'employer la Emile Basly au lieu de la rue Emile Basly. En polonais, les formules abrégées semblent plus courantes et couvrir un éventail plus vaste de désignations ${ }^{21}$. Cette idée est partagée par Bochnakowa : "En français l'omission de rue est exclue, par contre, en polonais courant, l'absence de ulica est fréquente (mieszka na Sienkiewicza), et il est exceptionnel de dire, en français : allons aux Champs-Élysées, plusieurs cinémas des Champs-Élysées, avec l'omission $\mathrm{d}^{\prime}$ avenue ${ }^{22}$.

$\mathrm{Vu}$ que, dans la présente étude, nous nous concentrons sur le transfert des noms propres, nous présenterons les noms de rues sous une forme invariante (p.ex. Bracka; Żurawia), même si dans le texte source les occurrences contiennent des formes fléchies : poszedt Bracka ('il a pris la rue Bracka'), w stronę Brackiej ('en direction de la rue Bracka'); na Żurawiej ('dans la rue Żurawia'), maszerował Żurawia ('il prenait la rue Żurawia').

Dans le corpus analysé, nous avons observé que les noms propres de rues sont presque toujours transférés sans changement ou avec une modification gra-

\footnotetext{
20 J. Cook, Traduire Marseille..., p. 228.

21 L. Waleryszak, Quelques réflexions..., p. 120.

22 A. Bochnakowa, Parcourir la ville, en français et en polonais, [in:] Traduire la ville, J. Brzozowski (réd.), Kraków 2006, p. 12.
} 
phique, de même que les noms de quartiers. Cieślikowa constate que la forme originale des toponymes apporte de la mélodie et de l'exotisme au texte traduit $^{23}$. Selon Hejwowski, il est important que le récepteur, curieux du texte traduit, puisse retrouver les lieux d'actions sur le plan de la ville ${ }^{24}$.

$\mathrm{Si}$, dans le texte de départ, le nom de rue est précédé du nom commun catégorisateur ulica, il est toujours traduit par rue :

\begin{tabular}{|c|l|l|}
\hline (7) & \multicolumn{1}{|c|}{ Uwikłanie - Les Impliqués } & \multicolumn{1}{c|}{ Podpalacz - Pyromane } \\
\cline { 2 - 3 } & $\begin{array}{l}\text { ulica Karłowicza - rue Karowicz (2) } \\
\text { ulica Lazienkowska - rue Lazienkow- } \\
\text { ska } \\
\begin{array}{l}\text { ulica Pawińskiego - rue Pawinski } \\
\text { ulica Mokotowska - rue Mokotowska } \\
\text { ulica Stawki - rue Stawki }\end{array}\end{array}$ & $\begin{array}{l}\text { ulica Kanarkowa - rue Kanarkowa } \\
\text { nicka } \\
\text { ulica Puławska - rue Pulawska } \\
\text { ulica Targowa - rue Targowa } \\
\text { ulica Fieldorfa - rue Fieldorf }\end{array}$ \\
\hline
\end{tabular}

Dans les exemples cités ci-dessus, nous observons des modifications graphiques autres que la suppression des signes diacritiques polonais. De nouvelles lettres apparaissent (rue Plaskownicka), d'autres disparaissent (rue Karowicz). Ce sont probablement des fautes de frappe qui n'avaient pas été éliminées au cours de l'édition du texte traduit.

Dans le texte source, plusieurs noms de rue ne sont pas précédés du nom commun rue. L'ajout du nom commun catégorisateur dans le texte cible est considéré par Hejwowski comme l'une des techniques de traduction des noms propres $^{25}$.

\begin{tabular}{|c|c|c|}
\hline \multirow[t]{2}{*}{$(8)$} & Uwikłanie - Les Impliqués & Podpalacz - Pyromane \\
\hline & $\begin{array}{l}\text { Lazienkowska - rue Lazienkowska (15) } \\
\text { Nowy Świat - boulevard Nowy } \\
\text { Swiat (6) } \\
\text { Krucza - rue Krucza (5) } \\
\text { Hoża - avenue Hoza (2), avenues } \\
\text { Hoza } \\
\text { Marszałkowska - avenue Marszal- } \\
\text { kowska } \\
\text { Puławska - avenue Pulawska } \\
\text { Wybrzeże Szczecińskie - quai Szcze- } \\
\text { cinski } \\
\text { Żwirki i Wigury - avenue Zwirki- } \\
\text { i-wigury }\end{array}$ & $\begin{array}{l}\text { Szaserów - rue des Chasseurs (4) } \\
\text { Płaskowicka - rue Plaskowicka (2), rue } \\
\quad \text { Plaskownica } \\
\text { Wołoska - rue Woloska }\end{array}$ \\
\hline
\end{tabular}

23 A. Cieślikowa, Jak „ocalić w tłumaczeniu”..., s. 315.

24 K. Hejwowski, Kognitywno-komunikacyjna..., s. 100.

25 K. Hejwowski, Iluzja przekładu..., s. 176-179. 
Cependant, le traducteur du roman Uwiktanie change parfois le nom commun rue en boulevard, avenue ou quai. Il nous semble que ce sont la spécificité et la grandeur d'une rue qui peuvent être à l'origine de cette décision. Regardons trois exemples qui échappent aux règles adoptées par le traducteur. L'ajout du nom commun catégorisateur quai pour traduire (ulica) Wybrzeże Szczecińskie est justifié par la localisation de cette rue le long de la Vistule. En revanche, la réduction du nom composé Wybrzeże Szczecińskie au nom simple Szczecinski ne semble pas convenable, d'autant plus qu'un autre nom composé de rue (Żwirki $i$ Wigury) est transféré et en plus adapté, avec les traits d'union, aux normes d'écriture des urbanonymes composés en français (rue du Chat-qui-Pêche, avenue Champs-Élysées). Le seul nom de rue (Szaserów $\left.{ }^{26}\right)$ est traduit comme Chasseurs, la notion liée à l'histoire de France et donc connue des récepteurs français. Néanmoins, la traduction littérale est une technique qui reste en opposition à la tendance contemporaine selon laquelle il convient de transférer sans changement les noms de lieux à l'intérieur des villes ${ }^{27}$. De plus, la traduction d'un seul nom de rue, lorsque les autres sont transférés, peut perturber la cohérence du texte et désorienter le récepteur français car, selon son savoir, les noms de rue en Pologne devraient être exprimés en polonais ${ }^{28}$.

Dans le roman Podpalacz, quelques noms de rue, privés du nom commun rue dans le texte de départ, sont simplement transférés dans le texte d'arrivée. Le traducteur n'ajoute pas le nom commun rue, ce qui peut entraîner la confusion. La désignation Pulawska (dix occurences) est traduite neuf fois comme la Pulawska. Le lecteur français a la chance d'apprendre, d'après le contexte, que c'est une rue, pourtant cette formule elliptique semble inadéquate dans la langue française :

Przy samej Puławskiej postawiono kilka niewielkich biurowców. (Pod 20)

Plusieurs immeubles de bureaux avaient surgi le long de la Pulawska. (Pyr 22)

- Powinien pan wiedzieć, że mieszkała niedaleko. Po drugiej stronie Puławskiej. (Pod 94)

- Je dois vous dire qu'elle habitait tout près. De l'autre côté de la Pulawska. (Pyr 112)

Dans l'exemple cité ci-dessous, le nom de rue, précédé de la préposition $\grave{a}$, peut être confondu avec un nom de ville, d'autant plus que cette rue n'apparaît qu'une seule fois dans le texte :

\footnotetext{
26 Selon le dictionnaire online de la langue polonaise sjp.pwn.pl, szaser est un soldat appartenant à un corps de cavalerie légère pendant les guerres de Napoléon $\mathrm{I}^{\mathrm{er}}$.

27 N. Paprocka, Les tendances dans..., p. 222.

28 E. Skibińska, Nazwy własne..., s. 166.
} 
- Poszedt z kolegami do Club Rocka na Racławickiej. (Pod 67)

- Il est partii au club de rock, à Raclawicka. (Pyr 79)

Le traducteur remplace souvent le nom propre de rue par le nom commun de l'institution qui s'y trouve :

\begin{tabular}{|c|c|c|}
\hline (9) & \multicolumn{1}{|c|}{ Uwikłanie - Les Impliqués } & Podpalacz - Pyromane \\
\cline { 2 - 3 } & $\begin{array}{l}\text { Lazienkowska - cloître (6) (UK : lieu de } \\
\text { crime) } \\
\text { Krakowskie - parquet régional (2), la } \\
\text { régionale (3) } \\
\text { ulica Krakowskie Przedmieście - par- } \\
\text { quet régional } \\
\text { Oczki - institut médico-légal (4) } \\
\text { Krucza - parquet (2) (UK : lieu de tra- } \\
\text { vail du héros principal, procureur } \\
\text { Szacki) } \\
\text { Wilcza - commissariat, chez les flics } \\
\text { Koszykowa - bibliothèque } \\
\text { Wiejska - Parlement }\end{array}$ \\
\hline
\end{tabular}

Les cinq premiers exemples de rues (ci-dessus) constituent les sièges des lieux principaux de l'action, assez connus des habitants de Varsovie et apparaissent souvent dans le texte. Grâce à cette technique de traduction plusieurs noms propres sont supprimés dans le roman Uwikłanie. Observons quelques exemples en contexte :

Grzelka zapytała, czy jest coś nowego w sprawie zabójstwa na Łazienkowskiej, [...]. (Uwi 87)

Grzelka demanda s'il y avait du nouveau dans l'affaire du meurtre du cloître [...]. (Imp 135)

Zadzwonit jeszcze na Oczki, dowiedział się, że sekcja zaplanowana jest na środę na dziewiata rano, i wyszedt. (Uwi 33)

Avant de sortir, il passa un dernier coup de fil à l'institut médico-légal, pour apprendre que l'autopsie avait été programmée le mercredi matin à neuf heures. (Imp 51)

Podpiszę panu ten akt oskarżenia, wyślemy go na Krakowskie i zobaczymy, co dalej. (Uwi 158)

Je vais vous le signer, votre acte d'accusation. On l'enverra à la régionale et on verra bien ce qui arrive. (Imp 249)

- [...] Kto prowadzi śledztwo?

Igor skrzywił sie, zanim odpowiedziat.

- Na Wilczej Kuzniecow, na Kruczej Szacki. (Uwi 36)

"[...] Qui dirige l'enquête?» 
Igor grimaça avant de répondre.

"Chez les flics, Kuzniecov. Au parquet, Szacki. » (Imp 56)

Les deux derniers exemples de rues (Koszykowa, Wiejska) sont aussi des sièges des institutions connues à Varsovie. La décision du traducteur de les supprimer semble justifiée, d'autant plus que ces rues n'apparaissent qu'une seule fois dans le texte :

- Jadę z Wólki na Koszykowa, muszę coś sprawdzić w bibliotece. (Uwi 181)

- Je vais à la bibliothèque, je dois vérifier un truc. (Imp 286)

Półgłówki z Wiejskiej z kolei były pewne, że maja swoją prywatną armię do nękania przeciwników politycznych. (Uwi 108-9)

Et les écervelés du Parlement considéraient les procureurs comme leur armée personnelle destinée à harceler leurs adversaires politiques. (Imp 169)

Bien des noms propres de rues sont supprimés dans le texte cible grâce à la traduction des noms communs qui les accompagnent :

\begin{tabular}{|c|c|c|}
\hline (10) & \multicolumn{1}{|c|}{ Uwikłanie - Les Impliqués } & Podpalacz - Pyromane \\
\cline { 2 - 3 } & $\begin{array}{c}\text { na Miodową do Urzędu Miasta - } \\
\text { à l'hôtel de ville } \\
\text { w mieszkaniu na Mokotowskiej - dans } \\
\text { l'appartement } \\
\text { w szpitalu na Niekłańskiej - dans un } \\
\text { lit d'hôpital } \\
\text { z instytutu na Sobieskiego - institut } \\
\text { médico-légal } \\
\text { dom na Żeromskiego - immeuble }\end{array}$ & \\
\hline
\end{tabular}

Dans le roman Uwikłanie, ces noms de rue sont présents une seule fois :

Szacki zaprowadził córkę do przedszkola, zawiózł Weronikę na Miodowa do Urzędu [...]. (Uwi 27)

Après avoir déposé sa fille à l'école, Teo Szacki avait conduit Weronika à l'hôtel de ville [...]. (Imp 43)

Byłem pierwszym oficerem przybyłym na miejsce, pojawiłem się w mieszkaniu na Mokotowskiej, kiedy był tam tylko nieświeży trup, jego siostra w katatonii i dwóch krawężników, [...]. (Uwi 220)

J'étais le premier officier parvenu sur place. Je suis arrivé dans l'appartement quand il n'y avait encore que le cadavre en décomposition, sa sour en état de choc et deux flics municipaux [...]. (Imp 345)

En revanche, dans le roman Podpalacz, nous trouvons plusieurs fois la désignation szpital na Szaserów ('hôpital rue Szaserów'). Cela permet au traducteur de supprimer quelques fois le nom propre Szaserów. Nous citons ci-dessous 
l'exemple où szpital na Szaserów est remplacé par l'hôpital militaire appelé d'ailleurs en polonais aussi szpital wojskowy.

Mortka od razu zadzwonit do szpitala na Szaserów. Odebrał jakiś doktor Maćkowiak. (Pod 55)

Mortka appela aussitôt l'hôpital militaire. Un certain Dr Mackowiak lui répondit. (Pyr 65)

En ce qui concerne les urbanonymes polonais représentés par les noms de rues (aussi par les noms d'avenues, de places, de ronds-points), il est nécessaire de mentionner ceux qui sont formés avec un nom propre de personne, notamment le nom de famille mis au génitif (p.ex. : plac Wilsona). Dans le texte cible, ces noms de famille prennent la forme du nominatif et ne sont pas précédés de la préposition de:

\begin{tabular}{|c|l|l|}
\hline (11) & \multicolumn{1}{|c|}{ Uwikłanie - Les Impliqués } & \multicolumn{1}{c|}{ Podpalacz - Pyromane } \\
\cline { 2 - 3 } & $\begin{array}{l}\text { Oczki - rue Oczka } \\
\text { Andersena - rue Andersen } \\
\text { Szymanowskiego - rue Szymanowski } \\
\begin{array}{l}\text { plac Piłsudskiego - place Pilsudski } \\
\text { plac Małachowskiego - square Mala- } \\
\text { chowski }\end{array}\end{array}$ & $\begin{array}{l}\text { ulica Generała Maczka - rue du Géné- } \\
\text { ral-Maczek } \\
\text { ulica Fieldorfa - rue Fieldorf } \\
\text { plac Dąbrowskiego - place Dabrowski } \\
\text { skwer Grotowskiego - square Grotow- } \\
\text { ski } \\
\text { point Dmowski }\end{array}$ \\
\hline
\end{tabular}

Dans le roman Uwikłanie, nous avons trouvé deux noms de rue qui échappent à cette règle :

$$
\begin{aligned}
& \text { Młota - rue Mlota (2) (forme au nominatif : Mlot) } \\
& \text { ulica Okrzei - rue Okrzei (forme au nominatif : Okrzeja) }
\end{aligned}
$$

Nous nous demandons si cette modification du génitif au nominatif est justifiée, cependant la langue française traite de la même façon les noms de voies allemandes en neutralisant ainsi les flexions casuelles ${ }^{29}$. Rappelons que l'une des techniques de traduction proposée par Hejwowski est le transfert avec l'adaptation au paradigme de flexion de la langue cible ${ }^{30}$. Puisqu'en français la flexion des noms polonais au génitif disparaît, nous parlons plutôt de la neutralisation de flexion et, dans la suite, nous allons ainsi appeler cette technique.

29 T. Grass, La traduction..., p. 668.
30 K. Hejwowski, Iluzja przekładu..., s. 176-179. 


\subsection{Avenues, allées, places et ronds-points}

Tous les noms d'avenues, d'allées, de places et de ronds-points se composent de deux segments dans les deux langues polonaise et française. Le nom commun catégorisateur est traduit (aleja, Aleje - avenue, allées ; plac - place, square ; rondo - rond-point). Quant au nom propre qui suit, nous n'avons trouvé que trois exemples de transfert sans changement :

\begin{tabular}{|c|c|c|}
\hline \multirow{2}{*}{$(12)$} & Uwikłanie - Les Impliqués & \multicolumn{1}{c|}{ Podpalacz - Pyromane } \\
\cline { 2 - 3 } & $\begin{array}{c}\text { Aleje Ujazdowskie - avenue Ujazdow- } \\
\text { skie } \\
\text { plac Bankowy - place Bankowy (3) }\end{array}$ & $\begin{array}{c}\text { Aleje Jerozolimskie - allées Jerozolim- } \\
\text { skie }\end{array}$ \\
\hline
\end{tabular}

La technique la plus souvent utilisée pour ce type d'urbanonymes est donc la traduction au moyen d'un équivalent lexical du nom propre :

\begin{tabular}{|c|c|c|}
\hline (13) & \multicolumn{1}{|c|}{ Uwikłanie - Les Impliqués } & \multicolumn{1}{c|}{ Podpalacz - Pyromane } \\
\cline { 2 - 3 } & $\begin{array}{c}\text { Aleje Jerozolimskie - allées de Jérusa- } \\
\text { lem Krakowska - avenue de Cracovie } \\
\text { plac Trzech Krzyży - place des Trois- } \\
\text { Croix (3) } \\
\text { plac Konstytucji - place de la Consti- } \\
\text { tution (2) } \\
\text { plac Zwycięstwa - place de la Victoire } \\
\text { rondo OnZ - rond-point des Nations- } \\
\text { rondo OnZ - rond-point des Nations- } \\
\text { Unies } \\
\text { Unies } \\
\text { rondo koło Rotundy - rond-point de la latraczna - rond-point du } \\
\text { Rotonde }\end{array}$ & \\
\hline
\end{tabular}

Waleryszak constate que lorsque le nom propre figure sous une forme adjectivale (Jerozolimskie, Bankowy), le traducteur devrait utiliser le complément de nom : de Jérusalem, de la Banque ${ }^{31}$. Il serait aussi préférable de traduire tous les noms propres d'allées et de places en vue de garder la cohérence (exemples (12) et (13)). Nous nous demandons encore pourquoi les noms propres d'allées, de places et de ronds-points sous une forme adjectivale sont d'habitude traduits (ex. (13)) et ceux de rues, qui sont pourtant du même type, ne le sont pas, p.ex. : rue Wilcza, rue Krucza, rue Belwederska, rue Kinowa, rue Poznanska. Ce manque d'uniformité constitue un problème déjà signalé par Lydia Waleryszak : 
[...] aucune œuvre étudiée ne présente de traduction uniforme. Il semblerait que les traducteurs fassent une distinction entre les noms de rues ou de places composés avec un adjectif et ceux qui le sont avec un ou plusieurs noms communs au génitif. Les premiers sont laissés tels quels (la rue Wiejska, la rue Wielka, la rue Smolna) alors que les autres sont entièrement traduits (la place des Trois-Croix, la place des Défilés, l'allée du 3-Mai). Seuls la rue des Jardins et la place du Château font exception alors qu'ils présentent, en polonais, une forme adjectivale. Le Robert illustré (2002) présente une désignation traduite partiellement (la place Zamkowy) ${ }^{32}$.

\section{Conclusion}

Nous constatons que les traducteurs suivent, en règle générale, les tendances contemporaines dans la traduction littéraire et préservent plutôt l'altérité. En revanche, le manque de cohérence dans le choix des techniques de traduction est visible. Pourtant, nous pouvons indiquer les techniques dominantes en fonction de la catégorie d'urbanonyme.

Tableau 1. Techniques dominantes de traduction d'urbanonymes polonais en français dans deux romans policiers

\begin{tabular}{|c|c|c|c|}
\hline $\begin{array}{c}\text { Catégorie } \\
\text { d'urbanonyme }\end{array}$ & Techniques de traduction dominantes & $\begin{array}{c}\text { Uwikłanie } \\
\text { Les Impliqués }\end{array}$ & $\begin{array}{l}\text { Podpalacz } \\
\text { Pyromane }\end{array}$ \\
\hline (1) quartier & $\begin{array}{l}\text { 1) transfert simple ou avec une } \\
\text { modification graphique } \\
\text { 2) ajout du nom commun catégorisateur } \\
\text { 3) remplacement par l'hypéronyme }\end{array}$ & $\begin{array}{l}+ \\
+ \\
+\end{array}$ & $\begin{array}{l}+ \\
- \\
-\end{array}$ \\
\hline (2) rue & 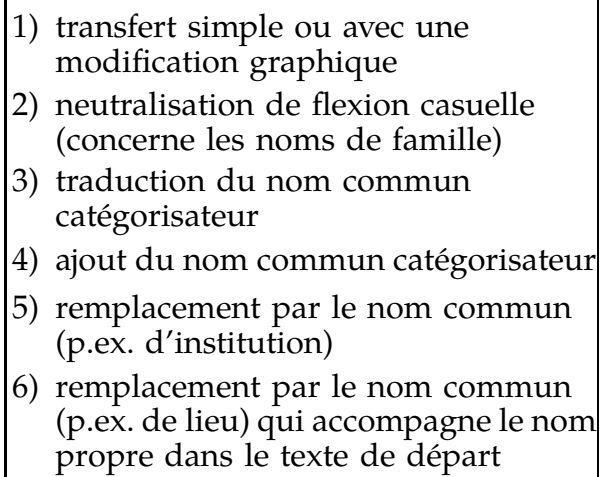 & $\begin{array}{l}+ \\
+ \\
+ \\
+ \\
+ \\
+\end{array}$ & $\begin{array}{l}+ \\
+ \\
+ \\
- \\
- \\
-\end{array}$ \\
\hline
\end{tabular}

32 Ibidem, p. 117. 


\begin{tabular}{|l|l|c|c|}
\hline $\begin{array}{c}\text { Catégorie } \\
\text { d'urbanonyme }\end{array}$ & Techniques de traduction dominantes & $\begin{array}{c}\text { Uwikłanie } \\
\text { Les Impliqués }\end{array}$ & $\begin{array}{c}\text { Podpalacz } \\
\text { Pyromane }\end{array}$ \\
\hline $\begin{array}{l}\text { (3) avenue } \\
\text { allée } \\
\text { place } \\
\text { rond-point }\end{array}$ & $\begin{array}{l}\text { 1) traduction litterale } \\
\text { traduction des noms communs } \\
\text { catégorisateurs }\end{array}$ & + & + \\
& $\begin{array}{l}\text { 3) transfert simple ou avec une } \\
\text { modification graphique } \\
\text { (concerne les noms de famille) } \\
\text { neutralisation de flexion casuelle } \\
\text { (concerne les noms de famille) }\end{array}$ & + & + \\
\hline
\end{tabular}

Les techniques les plus souvent utilisées pour les noms propres des catégories 1 et 2 sont le transfert simple (exemples (1)) et le transfert avec une modification graphique (ex. (2)). On les applique aussi pour la catégorie 3, mais seulement lorsque les noms propres contiennent un nom de famille. La modification graphique des noms propres résulte de la présence dans l'écriture polonaise des signes diacritiques inexistant dans la langue française $(t, o ́, s, c, q, e$, etc.). Leur suppression prive le récepteur français d'un certain indice d'étrangeté, par contre, la prononciation des noms propres pendant la lecture sera plus facile. Quant aux noms de famille composant les noms de rues, d'avenues, de places et de ronds-points, ils subissent une neutralisation flexionnelle et prennent la forme du nominatif (ex. (11)).

Contrairement aux noms propres des catégories 1 et 2 , ceux de la catégorie 3 sont le plus souvent traduits (ex. (13)). L'ajout du nom commun catégorisateur est une technique qui s'applique souvent pour les noms propres des catégories 1 et 2 (ex. (3) et (8)). Cela vient du fait que, dans la langue polonaise, nous avons la possibilité de ne pas mettre les mots osiedle ('quartier') et ulica ('rue') avant le nom propre. Grâce à la présence de ces noms communs catégorisateurs dans le texte cible, le lecteur français ne risque pas de se perdre dans cette multitude de lieux de Varsovie. Un grand nombre de noms propres de rues est tout de même précédé du mot ulica ('rue') dans le texte source. Ce nom commun, ainsi que les mots avenue, place et rond-point, sont toujours traduits (ex. (7) et (13)).

Il existe quelques techniques de traduction qui permettent la suppression de certains noms propres. Ces techniques s'avèrent utiles, lorsque le texte contient un grand nombre d'urbanonymes, comme le roman Uwikłanie. Le traducteur peut donc remplacer certains noms de rues par le nom commun de l'institution qui a son siège dans la rue indiquée (ex. (9)) ou par le nom commun de lieu qui accompagne le nom de rue dans le texte de départ (ex. (10)). La troisième technique de ce type, le remplacement par l'hypéronyme, est ap- 
pliquée pour les noms de quartiers qui s'étendent sur un terrain limité. Afin de supprimer le nom propre, le traducteur utilise une désignation plus large de ce même terrain (ex. (6)). Le roman Podpalacz n'étant pas si riche en urbanonymes, son traducteur n'avait pas besoin de recourir à ces trois dernières techniques.

\section{Sources d'exemples}

Pod - Chmielarz W., Podpalacz, éd. Marginesy 2018.

Pyr - Chmielarz W., Pyromane, É. Veaux (trad.), éd. Agullo/Livre de Poche 2018.

Uwi - Miłoszewski Z., Uwikłanie, éd. W.A.B. 2014.

Imp - Miłoszewski Z., Les Impliqués, K. Barbarski (trad.), éd. Mirobole/Pocket 2015.

\section{Bibliographie}

Ballard M., Le nom propre en traduction, Paris 2001.

Bochnakowa A., Parcourir la ville, en français et en polonais, [in:] Traduire la ville, J. Brzozowski (réd.), Kraków 2006, p. 11-17.

Cieślikowa A., Jak „ocalić w tłumaczeniu” nazwy własne?, [w:] Między Oryginałem a Przekładem II. Przekład, jego tworzenie się $i$ wptyw, M. Filipowicz-Rudek, J. Konieczna-Twardzikowa (red.), Kraków 1996, s. 311-320.

Cook J., Traduire Marseille : les repères spatiaux dans la traduction polonaise du roman Total Khéops de Jean-Claude Izzo, [in:] Challenges in Translation: Space, Culture and Linguistic Identity, R. Vasilescu, E. Nicolescu, R. Ştefan, A. Rădulescu (réd.), New York 2012, p. 225-237.

Grass T., La traduction comme appropriation : le cas des toponymes étrangers, "Meta » 2006, vol. 51(4), p. 660-670.

Handke K., Nazewnictwo miejskie, [w:] Polskie nazwy własne. Encyklopedia, E. Rzetelska-Feleszko (red.), Kraków 2005, s. 283-326.

Hejwowski K., Kognitywno-komunikacyjna teoria przekładu, Warszawa 2004.

Hejwowski K., Iluzja przekładu, Katowice 2015.

Lewicki R., Zagadnienia lingwistyki przekładu, Lublin 2017.

Newmark P., A Textbook of Translation, New York 1988.

Paprocka N., Les tendances dans la traduction des toponymes dans la littérature de jeunesse : l'exemple des traductions polonaises d'Alexandre Dumas, Les Trois Musquetaires, [in:] Challenges in Translation: Space, Culture and Linguistic Identity, R. Vasilescu, E. Nicolescu, R. Ştefan, A. Rădulescu (réd.), New York 2012, p. 212-224. 
Skibińska E., Nazwy własne we francuskim przekładzie Prawieku i innych czasów Olgi Tokarczuk, [w:] Przekładajac nieprzekładalne, W. Kubiński, O. Kubińska, T.Z. Wolański (red.), Gdańsk 2000, s. 157-169.

Słownik Języka Polskiego PWN, [online], https://sjp.pwn.pl/ , [accessible: 17.11.2020]. Vinay J.-P., Darbelnet J., Stylistique comparée du français et de l'anglais, Paris 1958.

Waleryszak L., Quelques réflexions sur la traduction des urbanonymes dans la littérature polonaise contemporaine, [in:] Traduire la ville, J. Brzozowski (réd.), Kraków 2006, p. 112-123. 